\title{
Back Radiation Suppression through a Semi- Transparent Round Ground Plane for a mm-Wave Monopole Antenna
}

\author{
Kirill Klionovski, Muhammad Fahad Farooqui, Atif Shamim \\ Integrated Microwave Packaging Antennas and Circuits Technology Laboratory \\ King Abdullah University of Science and Technology (KAUST) \\ Thuwal, Kingdom of Saudi Arabia \\ kirill.klionovski@kaust.edu.sa,muhammad.farooqui@kaust.edu.sa, atif.shamim@kaust.edu.sa
}

\begin{abstract}
Omnidirectional radiation pattern with minimum backward radiation is highly desirable for millimeter-wave telecommunication antennas. In this work, we propose a round, semitransparent ground plane of radius $0.8 \lambda$ with uniform impedance distribution that can reduce the back radiation of a monopole antenna by $8.8 \mathrm{~dB}$ as compared with a similar sized metallic ground plane. The value of uniform impedance is obtained through analytical optimization by using asymptotic expressions in the Kirchhoff approximation of the radiation pattern of a toroidal wave scattered by a round semitransparent ground plane. The semitransparent ground plane has been realized using a low-cost carbon paste on a Kapton film. Experimental results match closely with those of simulations and validate the overall concept.
\end{abstract}

Keywords-monopole antenna, ground plane, impedance, optimization, millimeter-waves.

\section{INTRODUCTION}

New millimeter-wave telecommunications require development of antennas which provide omnidirectional radiation pattern in upper half-sphere and low level of backward radiation. A monopole with a round ground plane is well-suited for these requirements. Different types of round ground planes [1], such as metallic ground planes, choke rings, impedance ground planes and semitransparent ground planes, have typically been used to reduce the backward radiation of a monopole. A metallic ground plane provides high suppression of the backward radiation but the radius of the ground plane must be large. Choke ring and impedance ground planes provide narrow-band solutions. A semitransparent ground plane of small radius has been used to obtain low backward radiation of a monopole for a wide bandwidth [1]-[3]. However, in those papers it was proposed to use ground planes with non-uniform isotropic resistive impedance which was realized through a complex R-card technology.

The proposed structures with non-uniform impedance distribution are difficult to realize for millimeter-wave bands, so in this paper we investigate the design and realization of a wideband monopole that employs a round semitransparent ground plane with uniform isotropic resistive impedance distribution. We optimize the value of the impedance to minimize the backward radiation of the monopole using an asymptotic formula for radiation pattern of a toroidal wave scattered by a round semitransparent ground plane. Also, we present experimental radiation patterns and $\mathrm{S}_{11}$ of the monopole with semitransparent and metallic ground planes.

\section{TheORETICAL Optimization Of THE TRAnSPARENCY OF THE GROUND PLANE}

Let's consider a monopole which is formed by a cylinder of radius $a$, length $L_{\mathrm{m}}$, and a conical base for increasing of the bandwidth. It is located on the axis of a semitransparent ground plane of radius $R$ and is excited by a coaxial cable

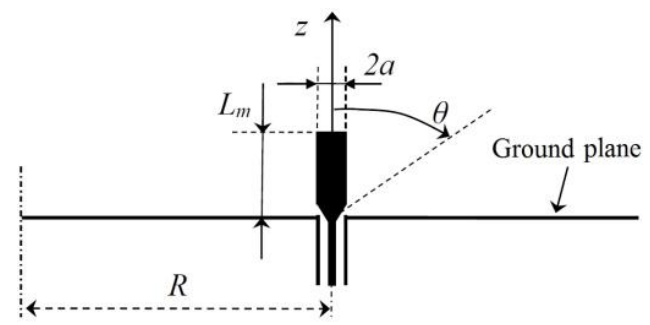

Fig. 1. The monopole with the ground plane. Side view.

(Fig. 1). The ground plane with isotropic transparency in cylindrical coordinates $(\rho, \varphi, z=0)$ is characterized by a reflection coefficient $\eta(\rho)$ and a transmission coefficient $\tau(\rho)=1-\eta(\rho)$ for a magnetic field intensity vector on the surface of ground plane. Impedance of semitransparent surface $Z$ is related to the reflection coefficient as $\eta(\rho)=60 \pi /(Z(\rho)+60 \pi)$. For our task, we assume that the ground plane has a central metallic area with radius of $R_{1}$ which is more than radius of the monopole, $Z(\rho)=0$ for $0 \leq \rho<R_{1}, a<R_{1}$, and a periphery area with a uniform isotropic resistive impedance $Z(\rho)=A$ for $R_{1} \leq \rho \leq R$. Let's optimize the parameters $A$ and $R_{1}$ of the ground plane to reduce the backward radiation of the monopole. For this case we consider a goal function which is mean square power radiated in the sector of angles $160^{\circ} \leq \theta \leq 180^{\circ}$ :

$$
P_{b}=\int_{8 \pi / 9}^{\pi}\left|H_{\varphi}^{\Sigma}(\theta)\right|^{2} d \theta \text {. }
$$


Here, $H_{\varphi}{ }^{\Sigma}(\theta)$ is the radiation pattern of the azimuthal component of the magnetic field intensity vector of the monopole with the ground plane in spherical coordinates $(r, \theta$, $\varphi)$. We use asymptotic formula for $H_{\varphi}{ }^{\Sigma}(\theta)$ in the Kirchhoff approximation which was obtained for the task of scattering of a toroidal wave by a round semitransparent ground plane [1] for minimization of the goal function (1):

$$
\begin{aligned}
& H_{\varphi}^{\Sigma}(\theta)=H_{\varphi}^{s}\left(R_{1}, \theta\right)+H_{\varphi}^{s}(R, \theta), \\
& H_{\varphi}^{s}(\rho, \theta)=\eta(\rho) H_{\varphi}(\arctan (h / \rho)+\pi / 2) \cos \theta \times \\
& \times\left[-2 J_{1}(k \rho \sin \theta)+i \sin \theta\left(J_{0}(k \rho \sin \theta)-\right.\right. \\
& \left.\left.-J_{2}(k \rho \sin \theta)\right) / \cos (\arctan (h / \rho))\right] e^{-i k \sqrt{\rho^{2}+h^{2}}},
\end{aligned}
$$

where $k=2 \pi / \lambda$ ( $\lambda$ is wavelength), $i$ is the imaginary unit, and $J_{n}(x)$ is Bessel function of order $n$ and argument $x$. We use the model of a monopole which was described in [1] to determine the radiation pattern of the monopole without the ground plane $H_{\varphi}(\theta)$. We assume that the electric current flows on the monopole surface along its axis and it can be presented as a set of annular electric currents $\mathbf{j}_{m}(\rho, z)=\delta(\rho-a) \delta\left(z-(m-1) L_{m} / M\right) \mathbf{z}_{0}[1]$ : $\mathbf{j}_{e}^{\Sigma}(\rho, z)=\sum_{m=1}^{M} \cos (0.5(m-1) \pi / M) \mathbf{j}_{m}(\rho, z)$, where $\delta(z)$ is the Dirac delta-function of argument $z ; \mathbf{z}_{0}$ is the unit vector in the $z$-direction. We seek the asymptotic expression for the radiation pattern of the monopole with the ground plane as a superposition of radiation patterns created by each annular electric current of the set $\mathbf{j}_{e}{ }^{\Sigma}(\rho, z)$ with the ground plane. The radiation pattern of the current $\mathbf{j}_{m}(\rho, z)$ in free space has the form $H_{\varphi}(\theta)=\sin \theta J_{0}(k a \sin \theta)$. After substituting $H_{\varphi}(\theta)$ for each current $\mathbf{j}_{m}(\rho, z)$ of the set $\mathbf{j}_{e}{ }^{\Sigma}(\rho, z)$ in (2) with the parameters $R=0.8 \lambda, a=0.05 \lambda, h=(m-1) L_{m} / M, L_{m}=0.25 \lambda, M=3$ and providing the minimization of the goal function (1) using the nonlinear conjugate gradient method, we obtain the next optimum value of the amplitude of impedance and the radius of metallization: $A=127 \mathrm{Ohm}, R_{1}=0.3 \lambda$.

\section{ThE PROTOTYPE AND EXPERIMENTAL RESUltS}

Based on the results of the optimization, a monopole with a round semitransparent as well as metallic ground plane of radius $8 \mathrm{~mm}$ was fabricated and measured. The monopole in the form of cylinder with radius of $0.4 \mathrm{~mm}$ (Fig. 2) has the total length of $2.4 \mathrm{~mm}$, where the conical base has a radius $0.25 \mathrm{~mm}$ and a length of $1.3 \mathrm{~mm}$. The monopole is excited by a $50-\Omega$ SMA connector. The semitransparent ground plane was fabricated through spreading of a thin layer of carbon paste with uniform thickness on a thin Kapton film. The value of the impedance is $129 \mathrm{Ohm}$ and the inner radius of the

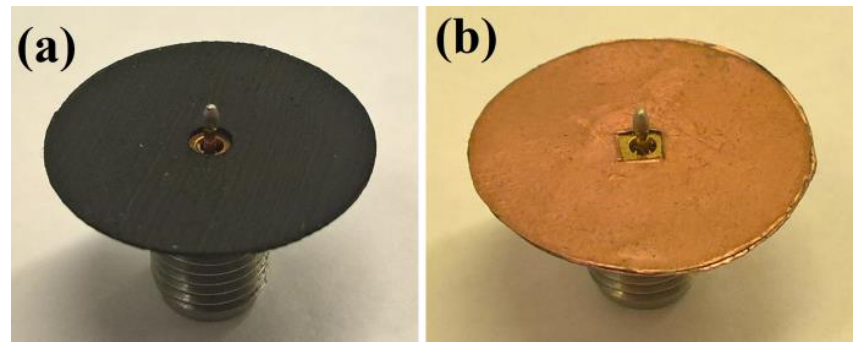

Fig. 2. Photo of the monopole with the semitransparent (a) and metallic (b) ground plane.

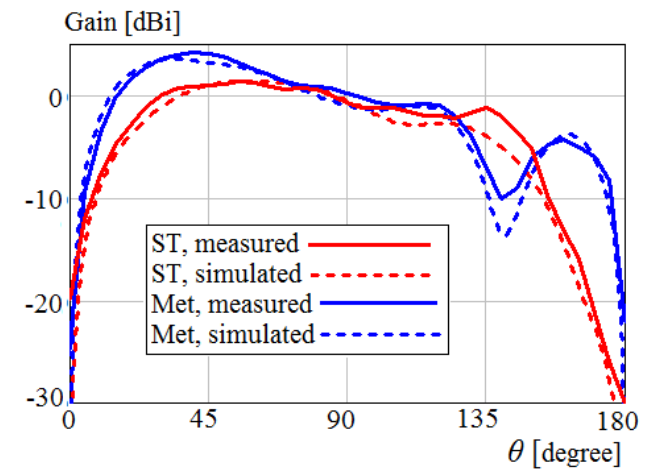

Fig. 3. Radiation patterns of the monopole with the ground planes.

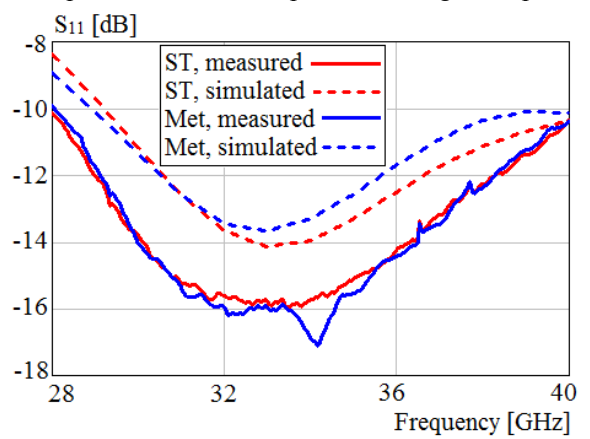

Fig. 4. $\mathrm{S}_{11}$ of the monopole with the ground planes.

semitransparent surface is $3 \mathrm{~mm}$.

Figs. 3, 4 plot the measured and simulated radiation patterns at frequency of $30 \mathrm{GHz}$ and $\mathrm{S}_{11}$ of the monopole with the semitransparent (ST) and metallic (Met) ground plane. Simulations were done using Ansoft HFSS software for models with dimensions which are indicated above. Fig. 3 shows that gain at $\theta=160^{\circ}$ is -13.1 and $-4.3 \mathrm{dBi}$ for the semitransparent and metallic ground plane's cases respectively. Also we can see the gain dropping in the upper half-sphere due to energy losses in the resistive layer of the semitransparent ground plane. Fig. 4 shows that the $S_{11}$ for the monopole with the semitransparent and metallic ground plane is similar.

\section{CONCLUSIONS}

Thus, using the model of the scattering of a toroidal wave by a round, semitransparent ground plane for the optimization of transparency of the ground plane has allowed us to determine the value of uniform isotropic resistive impedance. This design provides with approximately $9 \mathrm{dBs}$ lower back radiation from the semi-transparent ground plane as compared to the metallic ground plane.

\section{REFERENCES}

[1] V. Kaloshin and K. Klionovski, "On Radiation of Omnidirectional Axisymmetric Antennas with Circular Ground Planes," Journal of Communications Technology and Electronics, vol. 60, no. 10, pp. 10621071, Oct. 2015.

[2] R. W. Wang and V. V. Liepa, "Reduction of the edge diffraction of a circular ground plane by using resistive edge loading," in Antennas Propag. Soc. Int. Symp. Dig., vol. 2, 1985, pp. 769-771.

[3] R. W. Wang, "Reduction of the edge diffraction of a circular ground plane by using resistive edge loading," Ph.D. dissertation, Dept. Elect. Eng., Michigan Univ., Ann Arbor, MI, 1985. 